\title{
Analysis of Image Enhancement Techniques Used in Remote Sensing Satellite Imagery
}

\author{
Kriti Bajpai \\ MTech Student, \\ Department of Computer Science Engineering, \\ Gyan Ganga Institute of Technology \& Sciences, \\ Jabalpur (MP)
}

\begin{abstract}
Satellite Imagery is used in various research domains. These images contain major quality issues. However, it can be improvised by image enhancement algorithms in terms of contrast, brightness, feature reduction from noise contents, etc. These algorithms are employed to focus, sharp or smooth image to exhibit and examine the image attributes. Hence, the objective of image enhancement depends on the precise application. The objective of this paper is to provide brief information about the image enhancement techniques; which fetches progressive and optimum results for remote-sensing satellite imagery. To achieve this end, we perform various image enhancement algorithms, which are popular in present scenario to boost the quality of the images in several application areas of image processing.
\end{abstract}

\section{Keywords}

Remote Sensing, Satellite Imagery, Image Enhancement, Resolution, Spatial Domain, Frequency Domain, and Color enhancement.

\section{INTRODUCTION}

Satellite is a simulated structure which is situated in orbit to explore the planets for miscellaneous applications like weather, climate, geographical changes, monitoring network and broadcasting, etc. Satellite image is used to give a pleasant description of what is occurring at every location in the Earth, exclusively over the tropical oceans where statistics are unexpectedly appearing [1]. Data can only be immersed in specific locations in all over the localities throughout the world. However, short of this data forecasting would be precisely as challenging as not having satellites. The most important motive of our work is to concentrate on the image enhancement techniques in satellite imagery; as it emphasizes or improves image features such as edges, boundaries, or contrasts to assemble a graphic demonstration. This is more helpful for exhibiting and examining image attributes. The enhancement doesn't boost the built-in information and capacity of the data; it creates the dynamic scope of the chosen properties that can be explored effortlessly. Enhancing the image in visual perception is not only the motive of these techniques. Enhancement means simply identifying the real and the virtual objects. These objects are visible in satellite imagery, but sometimes do not appear due to shadow. These shadows are formed because of trees, buildings, mountains and flying objects such as airplanes and clouds. They hide some things in their shadows; our work is to identify these hidden objects within imagery. In hypothetical terms, the information enclosed in imagery can be described as

Image data $=$ regional + local pattern + noise $=$ background + foreground + noise $=$ low frequency + high frequencies +noise. [1] Noises can be either arbitrary or infrequent.

\author{
Rishi Soni \\ Assistant Professor, \\ Department of Computer Science Engineering, \\ Gyan Ganga Institute of Technology \& Sciences, \\ Jabalpur (MP)
}

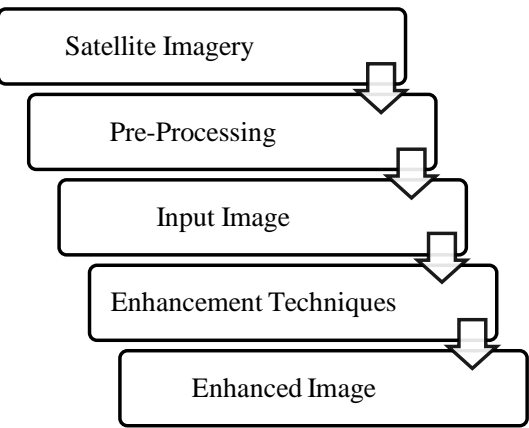

Fig 1: Process Diagram

In this paper, we implement the Image Enhancement techniques, and its application realm is satellite imagery. Image enhancement techniques are divided into three broad categories:

1. Spatial Domain Method,

2. Frequency-Domain Methods, and

3. Color Enhancement.

Numerous research papers are fully focused only on the first two methods of enhancement. However, sometimes we need to transform gray level image into the RGB color image; eventually, we have to consider the color enhancement of the images too. In the spatial domain, the image is filtered via pixel to pixel, and further in the frequency domain, it converts the image into a signal and later applies discrete transforms i.e. either Fournier, Laplacian or wavelet transforms.

\section{LITERATURE REVIEW}

The selection of enhancement techniques depends on certain features such as: specific task, image content, observer characteristics, and observing circumstances [2]. To understand the remote-sensing satellite imagery, we don't need any special knowledge of geology, ecology, oceanography, or any other branch of Earth science [1]. Firstly, we need to concentrate on the type of image enhancement techniques. Basically it is divided into two broad categories': First one is the spatial domain method- this method directly deals with pixel manipulation; and the second one is the frequency domain method [3]. The frequency domain method decomposes the image into a signal via Fourier transforms [4]. After that it instantly inverses the transform to get the desired image as a result. Fourier transforms are effortlessly flexible. In Fourier's analysis, the signal is converted into sine or cosine waves in different amplitude of frequency. Wavelet transforms also convert the signal but in scaled and translated version of mother wavelet that is Haar, Daubechies, Morlet, Mexican-hat, and Shannon Wavelet [5] [6]. High frequency inserts noise into the image which decreases the image quality. The Low-Pass filter will smooth the image and reduce noise. Low-pass filter and high- 
pass filter are in both the domains and work according to the domain's functioning procedure. High-pass filters are used for edge detection of the given image. It works by detecting interruptions in luminosity [4].

Contrast indicates the reflection of the dark zones within images [7]. Liner Contrast Stretch is the fundamental contrast enhancement technique. It increases the brightness level of the colored region and composes more recognizable elements of the image. Histogram Equalization is used to enhance the contrast within an image; it is considered as the most conventional enhancement algorithm. A histogram of the image represents the frequency of occurrence of all the gray level to the image [8]. Contrast enhancement is also done by Gaussian stretch; it increases the clarity of the image by a normal distribution method [1].

Color Enhancement Techniques are HSI transform \& Pseudo color image. HIS transform maps the RGB images into HSI colored image. Pseudo-colors map the one-dimensional gray scale image into an intense RGB color spectrum [9]. It doesn't boost the image information; but it can set some details more visible by increasing space between successive gray levels [10].

\section{IMPLEMANTATION \& RESULTS}

\subsection{Preprocessing}

To begin implementation, we need the image dataset. Which we get from the QuickBird open source satellite imagery data set. We are exploring the satellite imagery that belongs to Kolkata city West Bengal 02NOV21044347-M2AS_R1C1000000185940_01_P001.TIF for implementation. In the direction of examining an enormous image, we are using ENVI 5.0 software. ENVI GUI contains numerous functionality windows, i.e. support to analyze imagery. It helps us to make sub-images by clipping the image from the imagery. After getting the images, we move forward for the next phase that is implementations of image enhancement techniques via MATLAB 9.2 tool [11]. Here an actual procedure was performed to manipulate the image matrices.

For Implementation, we have used the clip of satellite imagery in the RGB image form which is as follows: Clip 1 is used to implement Spatial Domain in a low-pass filter, high-pass filter, and the color enhancement methodology. Clip 2 is used to implement frequency domain in Fourier transforms filters. Clip 3 is used to implement Contrast Enhancement and clip 4 is used to implement 2D-DWT.

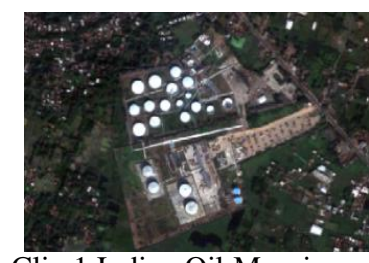

Clip 1 Indian Oil Mourigram Terminal

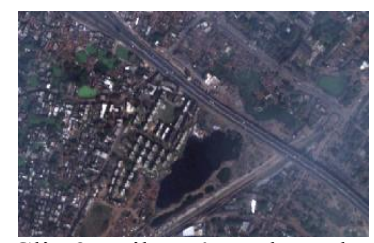

Clip 3 Railway's Duke Lake

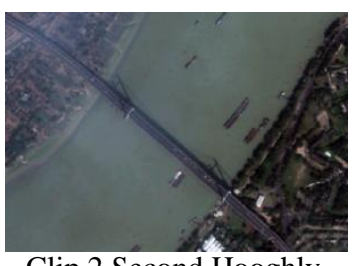

Clip 2 Second Hooghly Bridge

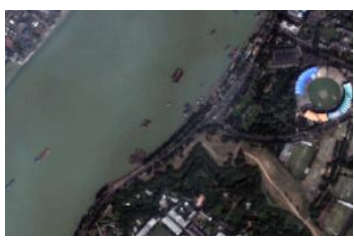

Clip 4 Eden Garden
Fig 2: Images used to implement Image Enhancement Techniques

\subsection{Image enhancement techniques implementations}

\subsubsection{Spatial domain}

Spatial Domain method mainly focuses on point to point manipulation of the image; as it goes in every single pixel intensity of the image. [12] [13]

The basic formula of this method:

$g(x, y)=T[f(x, y)]$

\subsubsection{Spatial low-pass filter}

The low - pass filter is known as smoothing or blurring filter. It was developed for making an image smooth and to reduce the noise from the image. High frequency initiates noise into an image that decreases the image quality. Low-pass filters are divided into three sub-filtering techniques:

1. Moving average filter,

2. Median Filter, and

3. adaptive filter.

MATLAB implementations of these techniques are shown in (Figures 2,3,4 \& 5).
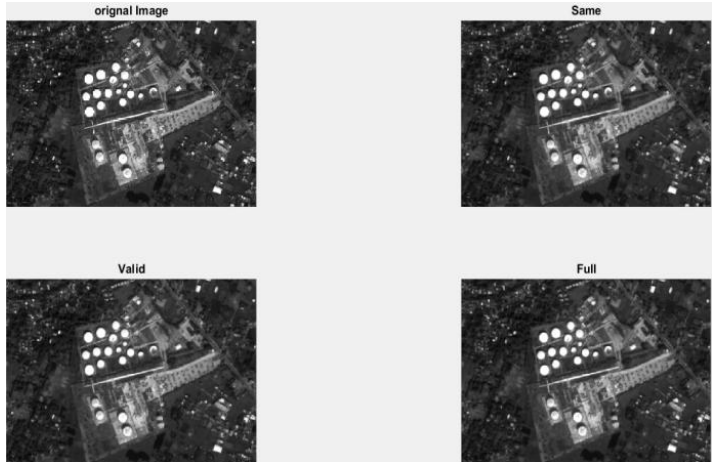

Fig 3: Spatial low-pass filter via built-in function

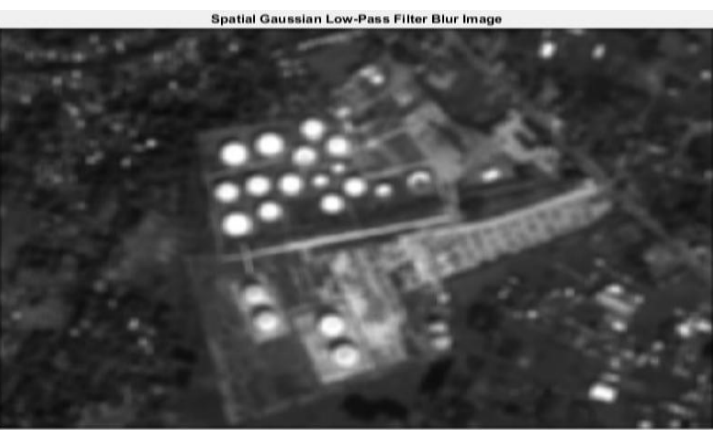

Fig 4: Blur Image via Low-Pass Filter

\subsection{Moving average filter}

Moving average filter replaces the data value by the average of the given data point and the specific number of pixel starts from the left to the right. [11]
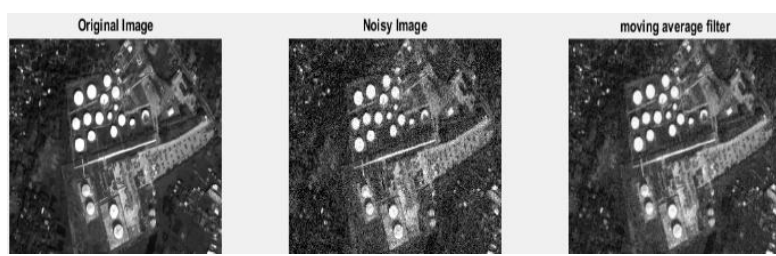

Fig 5: Moving average filter to reduce Gaussian noise 


\subsection{Median filter}

Median filter is commonly defined as the superscript of moving average filter. There are two causes for it: Firstly, median is a set of $n$ numbers where $n$ is an odd integer which is always one of the data standards existing in the set. Secondly, the median is less convoluted to error or to detect data consequence. [14] [15]
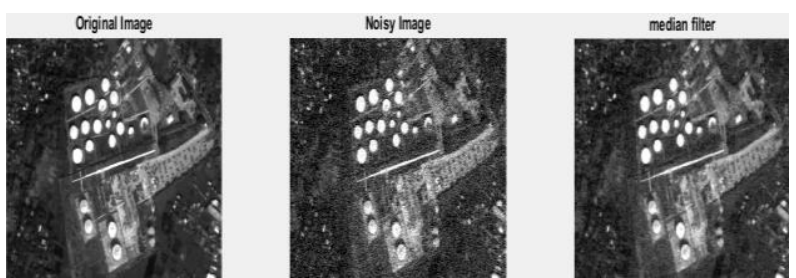

Fig 6: Median filter to remove Gaussian noise.

\subsection{Adaptive filter}

When the calculation is based on mean and variance of the gray levels, it is considering as the adaptive filter. [1]
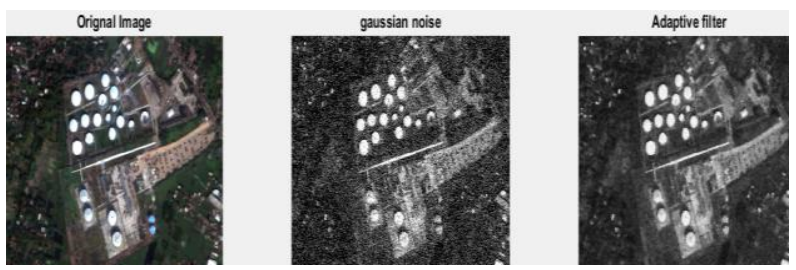

Fig 7: Adaptive filter to remove Gaussian noise

\subsubsection{Spatial high-pass filter}

Spatial high-pass filter might seem as de-blurring the image or sharpening the image. In remote sensing, two operations are more reliable for studying the image attributes: First, it is the arithmetic operation- Image subtraction method and second is also based on the mathematical concept of the derivative. The derivative of a continuous function is specified by the point ratio, i.e. the rate of change of that function value of particular point. [1]
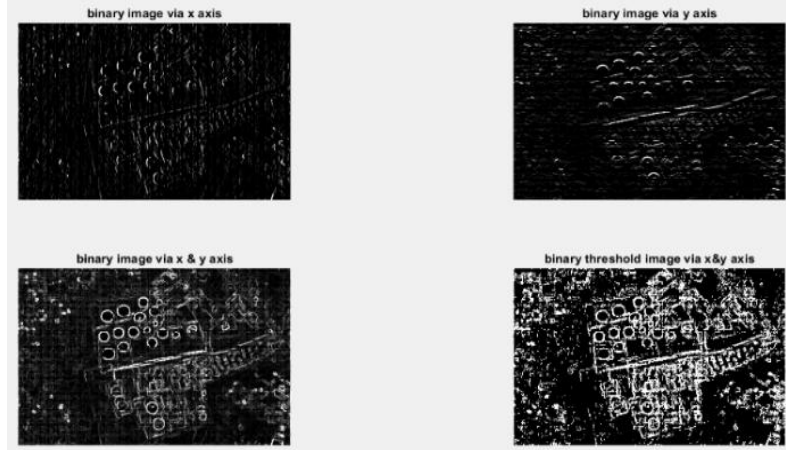

Fig 8: Spatial High-Pass Filter

\subsection{Image subtraction method}

An image can be computed by the sum of its low and highfrequency mechanism, plus noise. The low-frequency (LF) part can be partially computed using a low-pass filter. This LF image can be subtracted from the primary, unfiltered image; leaving behind the high-frequency component. The resulting image can be added back to the original, thus effectively doubling the high-frequency (HF) part; or a proportion of the low-pass filtered image can be subtracted from the creative image to give HF image. [1] [10] The concept of Thomas:

$R^{*}=R-f R^{\prime}+C$
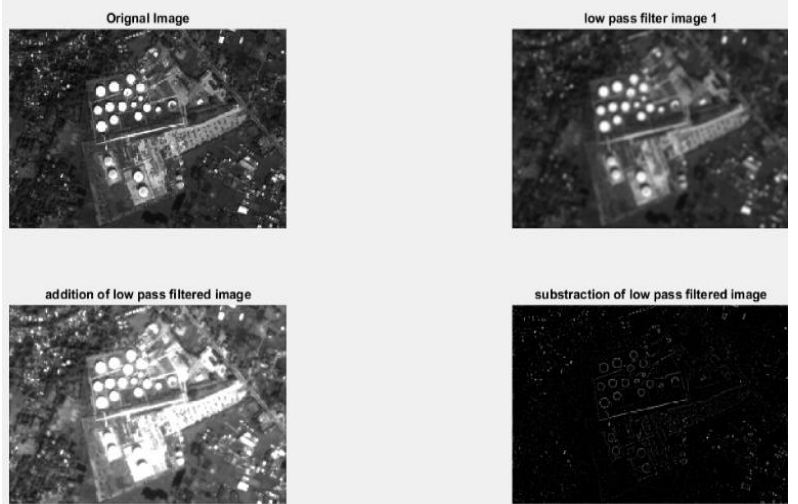

Fig 9: Image subtraction method for high pass filter

\subsection{Derivative-based Method}

The derivative of a continuous function is specified by the point ratio, i.e. the rate of change of that function value of particular point. For instance, the first derivative of position with respect to time is velocity. The greater the velocity of an object, the more rapidly it changes its point of view with respect to time. The velocity can be computed at any time after a signal is produced. As an alternative, derivatives are approximate in terms of differences between the values of neighboring pixels in the $x$ and $y$ directions, while oblique or angle differences are also used. [1]

$$
\begin{aligned}
& \Delta x p(i, j)=p(i, j)-p(i-1, j) \\
& \Delta y p(i, j)=p(i, j)-p(i, j-1)
\end{aligned}
$$
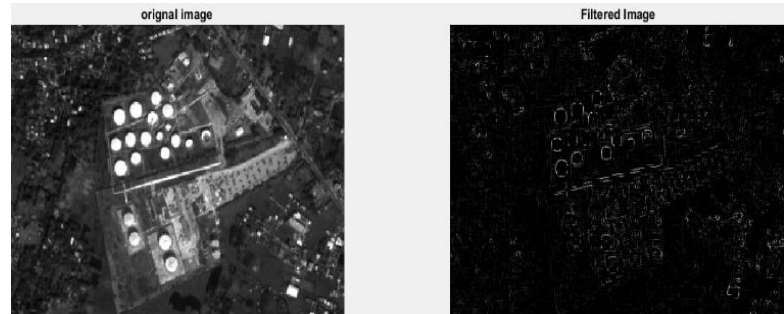

Fig 10: Derivative based high-pass filter

\subsubsection{Contrast enhancement}

Contrast shows the reflectiveness in the dark zones of the images. Contrast enhancement is one of the most important features of the image enhancement technique i.e. it manipulates the illumination level of the image. Its sub fields are linear contrast stretch, histogram equalization and Gaussian stretch respectively. [7] [16] [17]

\subsection{Linear contrast stretch}

The motivation behind the contrast stretching is to boost the dynamic ranges of the gray levels within the image being processed. This is the concept of adjusting the dynamic range of the gray level of the image. LCS is the straightforward contrast stretching algorithm that stretches the pixel values of the low-contrast image or high-contrast image by multiplying the dynamic range across the whole image spectrum from 0- (L-1) [13]. Contrast stretch is done by scaling all the pixel of an image by a constant $\mathrm{k}$. The basic formula of this method:

$g[m, n]=f[m, n] * k$

Adjusting the contrast of image is the modifications of the ranges of luminance values present in an image. 


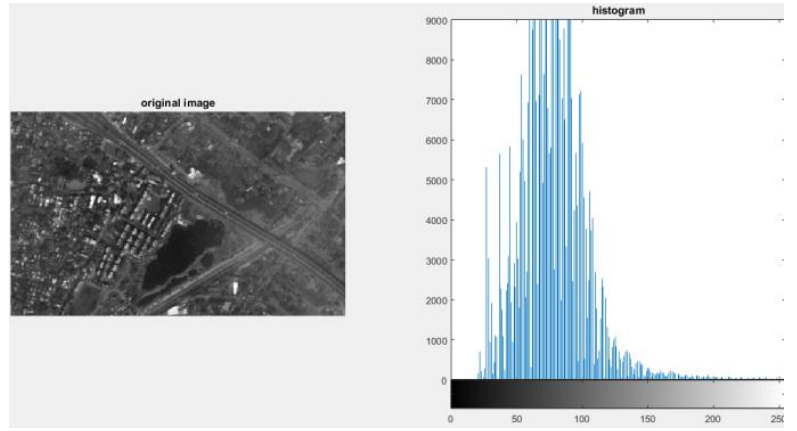

Fig 11: Original image histogram
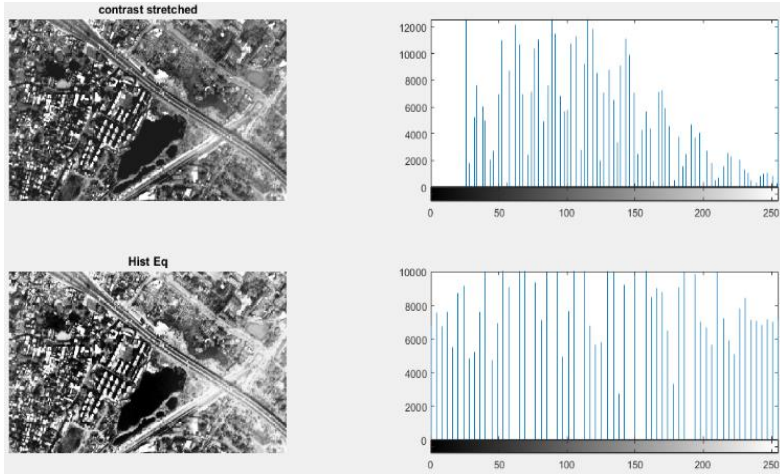

Fig 12: Linear contrast stretch histogram

\subsection{Histogram equalization}

Histogram equalization is a technique for adjusting the image contrast. Histogram simply plots the frequency at which each gray level occurs from 0 to 255 , and then the intensity values of the original image are plotted to give a uniform histogram of intensity values. Let $f$ be a given image signified as a $m_{r}$ by $m_{c}$ matrix of integer pixel concentrations ranging from 0 to $\mathrm{L}-1$. $\mathrm{L}$ is the number of possible intensity standards, 255 [7] [8] [13]. Let $p$ denote the normalized histogram of $f$ with a bin for each possible intensity values.

\section{$p_{n}=\frac{\text { number of pixels with intensity } n}{\text { total number of pixels }}$}

Where $\mathrm{n}=0,1, \ldots, \mathrm{L}-1$.
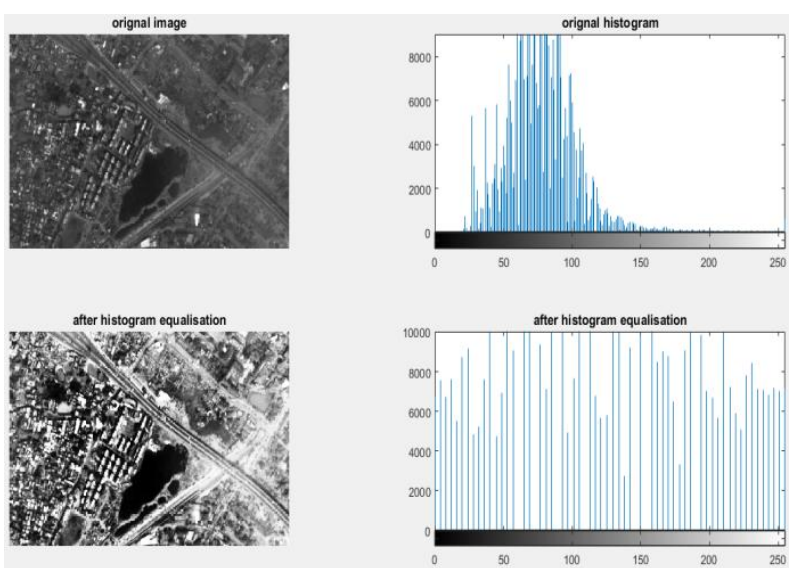

Fig 13: Gray image histogram equalization

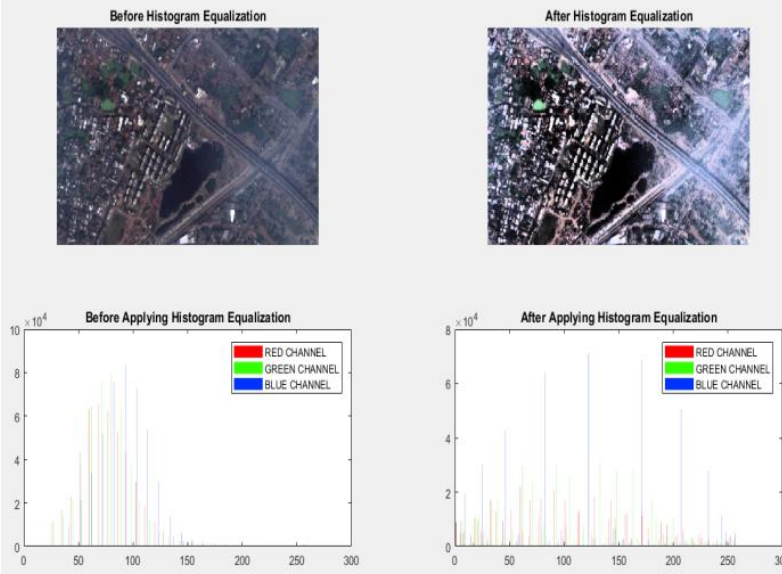

Fig 14: RGB color image histogram equalization

\subsection{Gray level slicing}

Gray level slicing is the spatial domain methodology which approximately works as band-pass filtering. The motive of the gray level slicing is to emphasis a range of gray values of the image. [10]

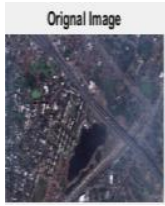

4

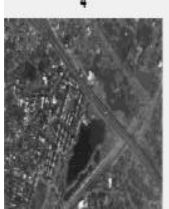

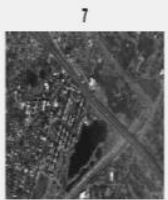

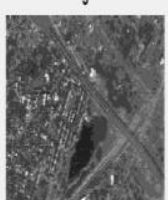

Fig 15: Gray Level Slicing

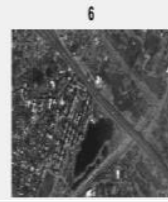

2

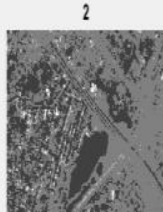

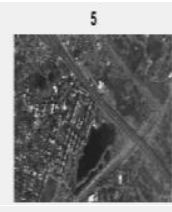

1

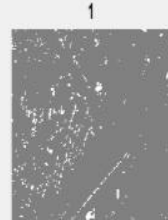

3.2.1.3.4 Gaussian stretch (normal distribution)

A contrast-enhancement based on the histogram of the pixel values is sent for a Gaussian stretch because it holds the filtering of the observed histogram to a Normal or Gaussian histogram. A Normal distribution gives the chance of observing a value $\mathrm{x}$ given the mean $\overline{\mathrm{x}}$ is defined by: [1]

$p(x)=\frac{1}{\sigma \sqrt{2 \pi}} e^{-\frac{(x-\bar{x})^{2}}{2 \sigma^{2}}}$

The standard deviation, $\sigma$, is defined as the range of the variable for function $p(x)$ [1] [18]
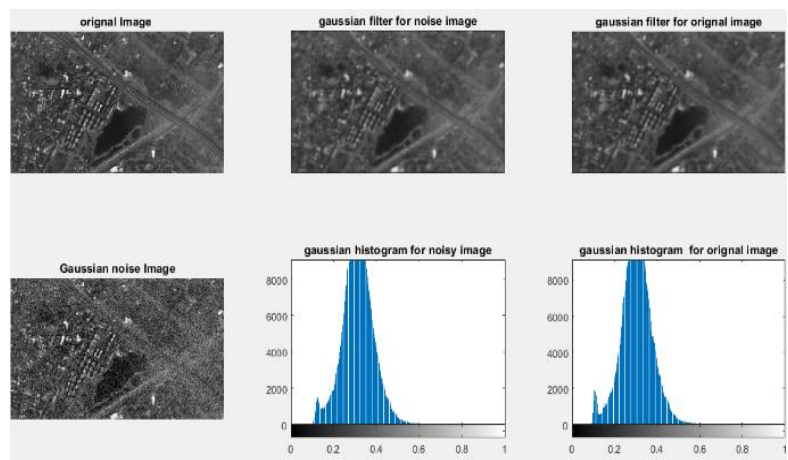

Fig 16: Gaussian stretch via Gaussian filter 


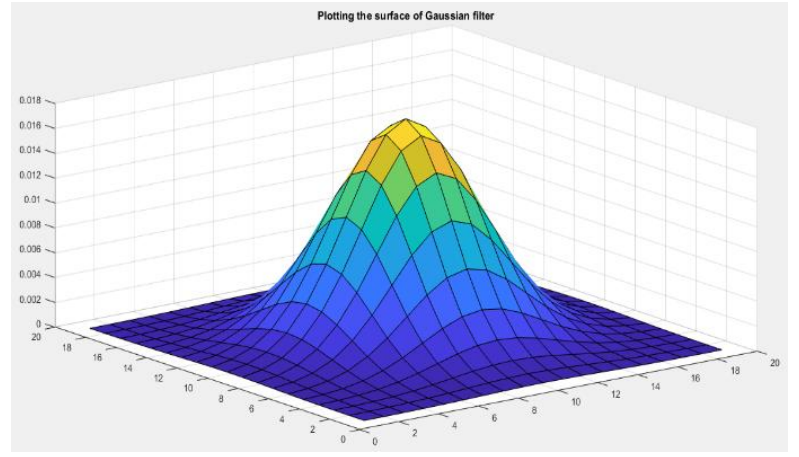

Fig 17: Plotting Gaussian filter

\subsubsection{Frequency domain}

Frequency refers to the rate of recurrence of some periodic event. A modulating signal can be transmuted into a serialized of simple cyclic variations. The Fourier transforms is used to decay a signal from a set of sine waves from various aspects like frequency and phase. The technique of quick frequency inclusion or execution is termed as frequency domain filtering. Spatial domain filtering is adept by convolving the image with the filter kernel. [10]

Convolution in spatial domain=Multiplication in frequency domain.

In the frequency domain, filtering relates to the multiplication of the image spectrum by the Fourier Transform of the filter kernel which is symbolized as the frequency response for the filter. [4]

Filtering in Frequency domain:

$G(k, l)=F(k, l) \times H(k, l)$

\subsubsection{Discrete fourier transform}

The DFT of a two-dimensional image can be visualized by displaying the spectrum of the image's component frequencies [18]. Image is an independently varying function. It is one way to analyse spatial function variations. This is done to decompose an image into a set of orthogonal functions. Fourier transformed functions are used to transform the intensity within the image into the domain of spatial frequency [6].

$F(u, v)=\sum_{x=0}^{M-1} \sum_{y=0}^{N-1} f(x . y) e^{-i 2 \pi\left(\frac{u x}{M}+\frac{v y}{N}\right)}$

The concept behind the Fourier transforms is that any waveform can be constructed using a sum of sine and cosine waves of different frequencies. The exponential in the above formula can be expanded into Sine and Cosines with the variables $\boldsymbol{u}$ and $\boldsymbol{v}$ determining these frequencies. The inverse of the above discrete Fourier transforms is given by the following equation:

$f(x, y)=\frac{1}{M N} \sum_{u=0}^{M-1} \sum_{v=0}^{N-1} F(u, v) e^{i 2 \pi\left(\frac{u x}{M}+\frac{v y}{N}\right)}$

Thus, if we have $F(u, v)$, we can obtain the corresponding image $(f(x, y))$ using the inverse discrete Fourier transform.

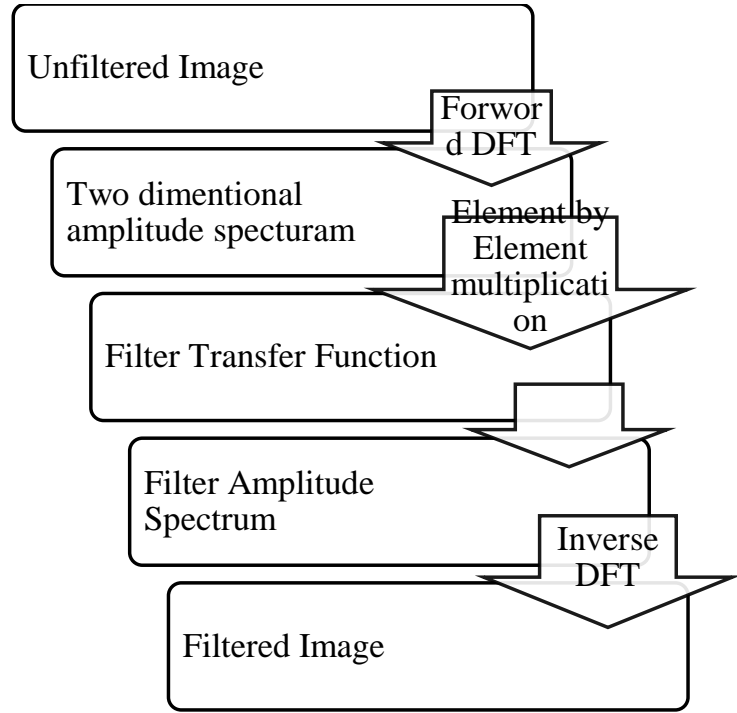

Fig 18: Steps in the frequency domain filtering.

\subsection{Low-pass filter (smoothing)}

The Low-Pass Filter averages out rapid changes in intensity. It attenuates the high frequencies and leaves the low frequencies of the Fourier transform relatively unchanged. It will smooth the image and reduce noise. It can be classified into two types:

1) Non-separable filter, and

2) Separable Filter

Non-separable filter transfer function:

$H(k, l)=\left\{\begin{array}{lr}1, \text { for } \sqrt{k^{2}+l^{2}} \leq D_{0} \\ 0, \quad \text { otherwise }\end{array}\right.$

Here, $D_{0}$ is the cutoff frequency of the low pass filter. The cutoff frequency governs the amount of frequency elements approved by the filters [10].

Separable Filter:

$H(k, l)= \begin{cases}1, \text { for } k \leq D_{k} & \text { and } l \leq D_{l} \\ 0, & \text { otherwise }\end{cases}$
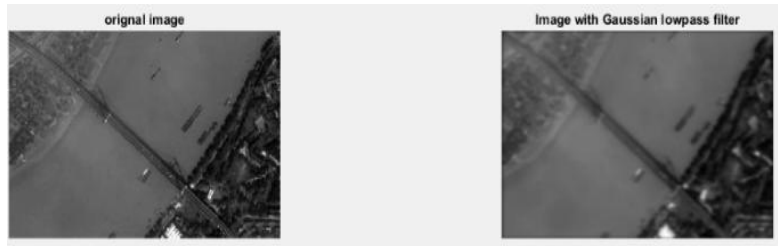

Fourier Spectrun

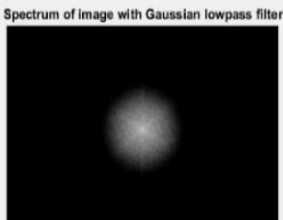

Fig 19: Low-pass filter\& its Fourier spectrum.

\subsection{Ideal low pass filter}

The 2D ideal low-pass filter (ILPF) is a filter whose transfer function satisfies this relation: [10]

$$
H(k, l)=\left\{\begin{array}{l}
1 \text { if } D(k, l) \leq D_{0} \\
0 \text { if } D(k, l)>D_{0}
\end{array}\right.
$$



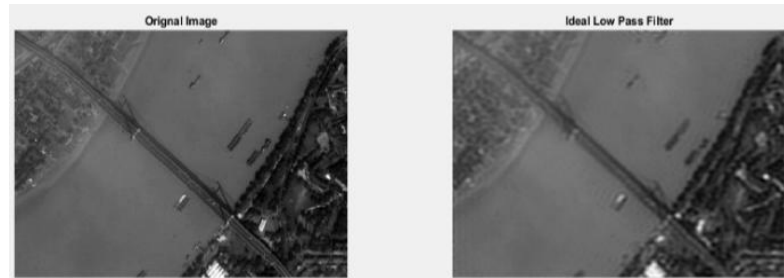

Fig 20: Ideal Low pass Filter

\subsection{Butterworth low-pass filter}

Butterworth low-pass filter (BLPF) is used to eliminate high frequency noise with minimum loss of signal elements. The assignment function of a BLPF of order $n$ with cut-off frequency $D_{0}$ is given by [4]

$$
H(k, l)=\frac{1}{1+\left[\sqrt{\frac{k^{2}+l^{2}}{D_{0}}}\right]^{2^{n}}}
$$

For this smooth transition filter, a cut-off frequency effort is chosen such that $H(k, l)$ it is a hold the percentage of its maximum cut-off.
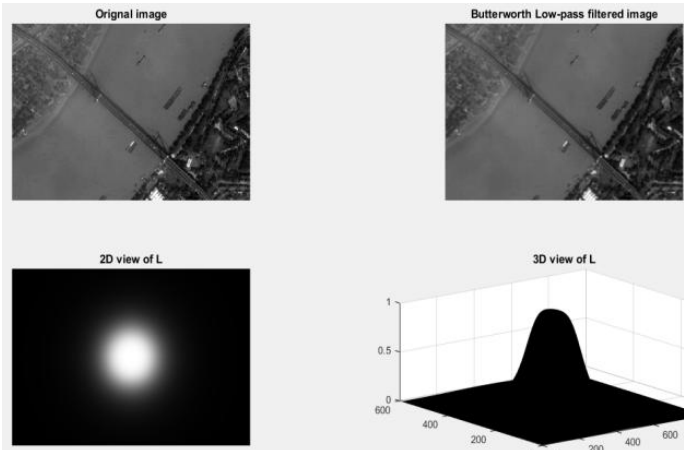

Fig 21: Butterworth low-pass filter with its 2D \& 3D surface plot.

\subsection{Gaussian low-pass filter}

Gaussian filters minimize the problem that occurred in an ideal filter. This problem is formally acknowledged as the ringing effect. Because at some point the transition between one color to the other cannot be defined precisely, due to which the ringing effect appears at that point. The assignment function of two-dimensional Gaussian Low-pass filter (GLPF) is specified by [4]

$H(k, l)=\frac{1}{\sqrt{2 \pi \sigma^{2}}} e^{-\frac{k^{2}}{2 \sigma^{2}}}$
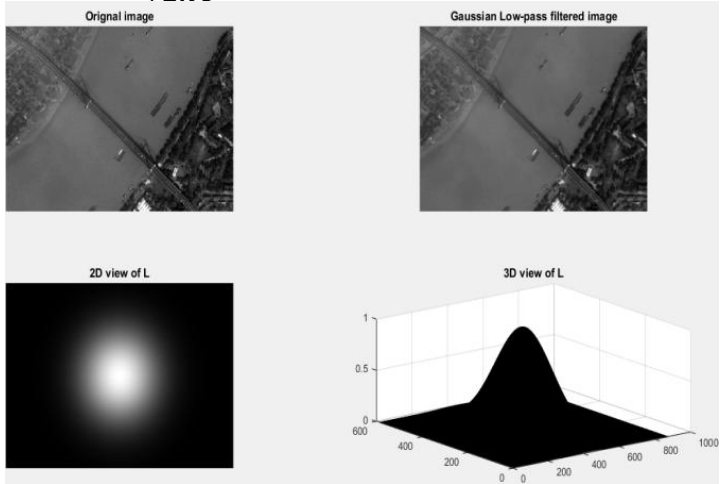

Fig 21: Gaussian low-pass filter with its 2D \&3D surface plot

\subsection{High-pass filter (sharping)}

Low pass and high pass filters always complement each other. The 2D ideal high-pass filter (IHPF) is a filter whose transfer function satisfies the following relation: [4]

$H(k, l)=\left\{\begin{array}{l}0, \text { if } D(k, l) \leq D_{0} \\ 1, \text { if } D(k, l)>D_{0}\end{array}\right.$

Ideal High pass Filter is not practically achievable.
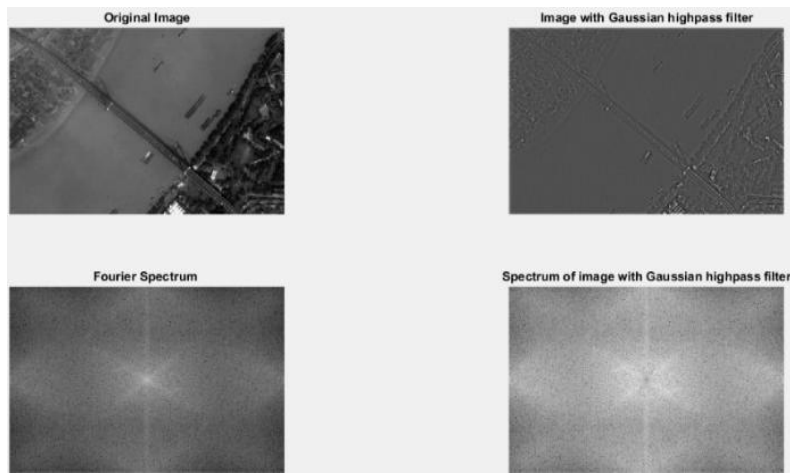

Fig 22: High pass filter \& its Fourier spectrum

\subsection{Butterworth high-pass filter}

The assignment function of Butterworth high-pass filter (BHPF) is specified by [4]

$$
H(k, l)=\frac{1}{1+\left[\frac{D_{0}}{\sqrt{k^{2}+l^{2}}}\right]^{2^{n}}}
$$
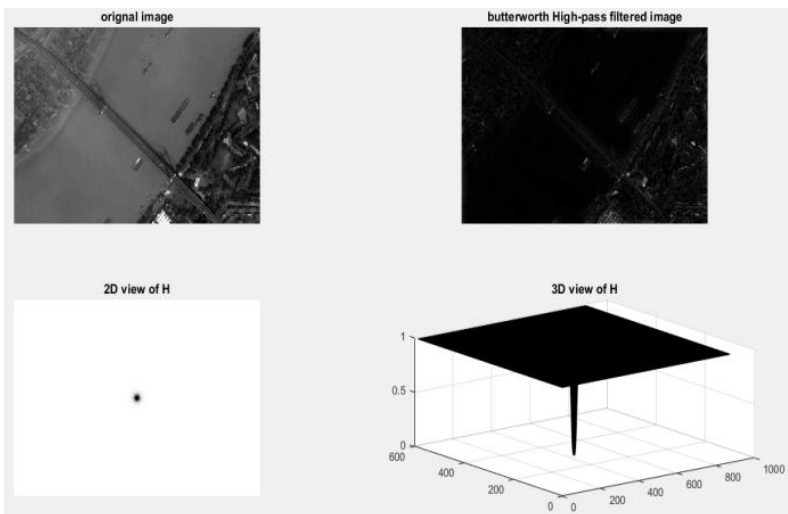

Fig 23: Butterworth high-pass filter with its 2D \& 3D surface plot

\subsection{Gaussian high-pass filter}

Gaussian filter is admired for the lack of calling up, and noise escaped objects. Gaussian high pass filter has the same concept as ideal high pass filter; but again the transition is smoother as compared to the ideal one. The assignment function of two-dimensional Gaussian Low-pass filter (GHPF) is specified by [4]

$H(k, l)=1-e^{-D^{2}(k, l) / 2 \sigma^{2}}$ 


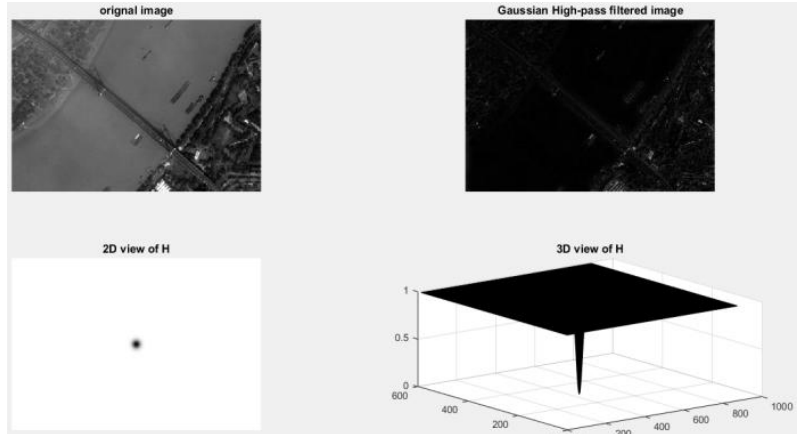

Fig 24: Gaussian high-pass filter with its 2D \&3D surface plot

\subsection{Notch filters (band-stop)}

Notch filters are known as band-stop filters. These filters are used to remove repetitive "Spectral" noise from an image. Notches are like a narrow high-pass filter, but the "notch" out frequencies other than the dc component decrease selected frequency and leave other frequencies of the Fourier transform comparatively unchanged. Repetitive noise in an image is sometimes seen as an optimistic peak somewhere other than the source. We can suppress such noise effectively by carefully erasing the peaks. One way to do this is to use a notch filter to remove that particular frequency from the image.
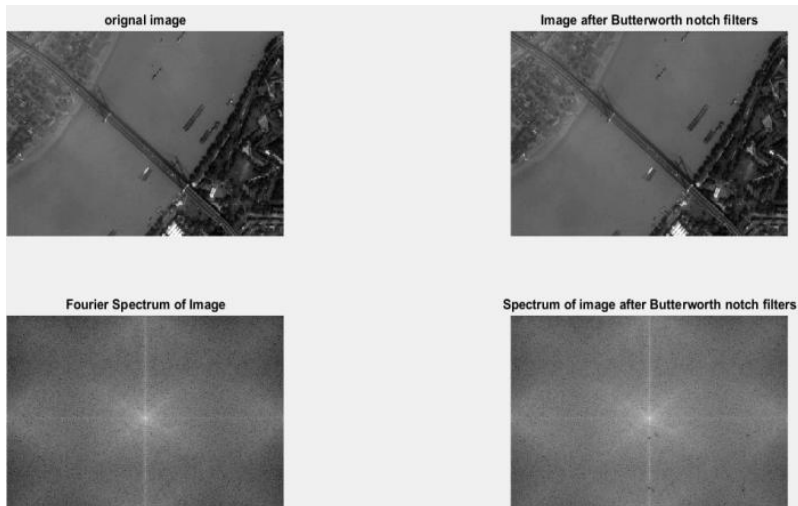

Fig 25: Notch Filter\& its Fourier spectrum

\subsubsection{Discrete wavelet transform}

The Discrete Wavelet transforms (DWT) is obtained by filtering the signal concluded by a series of digital filters at discrete scales. This scaling procedure is done by transforming the resolution of the signal by the method of substitute sampling. [19] [21]

The DWT can be computed by applying either convolution centered or lifting based techniques. In both approaches, the input order is decayed into low-pass \& high- pass sub bands. Each method depends on half number of samples in the original order.

\subsection{Two-dimensional discrete wavelet transform}

To implement the 2D DWT, we have used Haar Mother Wavelet. It is a bipolar step function. The expression of Haar wavelet is specified as a result of: [6] [18] [19]

$\psi(t)=\left\{\begin{array}{c}1 \text { when } 0<t<\frac{1}{2} \\ -1 \text { when } \frac{1}{2}<t<1 \\ 0 \quad \text { otherwise }\end{array}\right.$

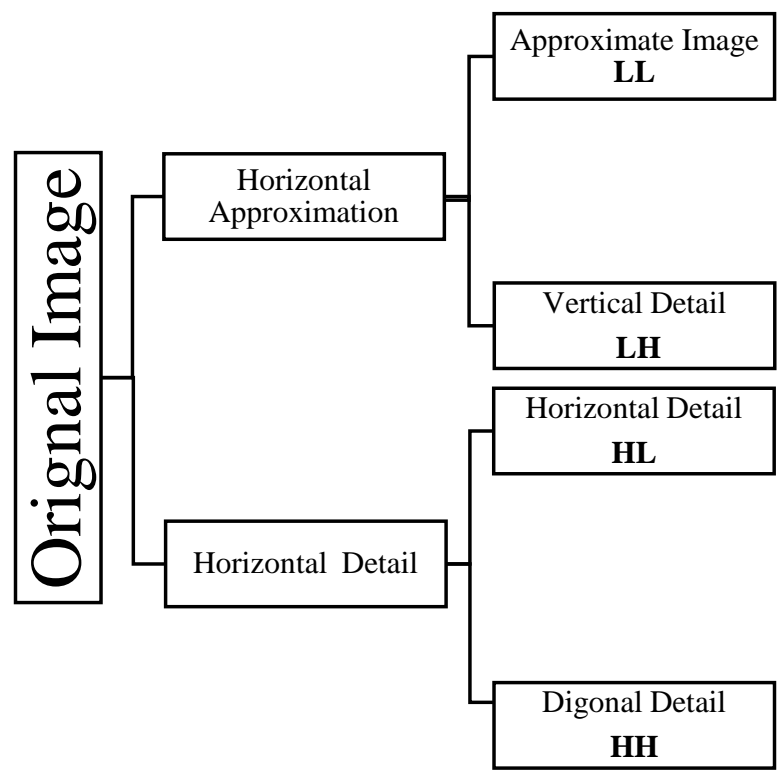

Fig 26: Discrete Wavelet Transform
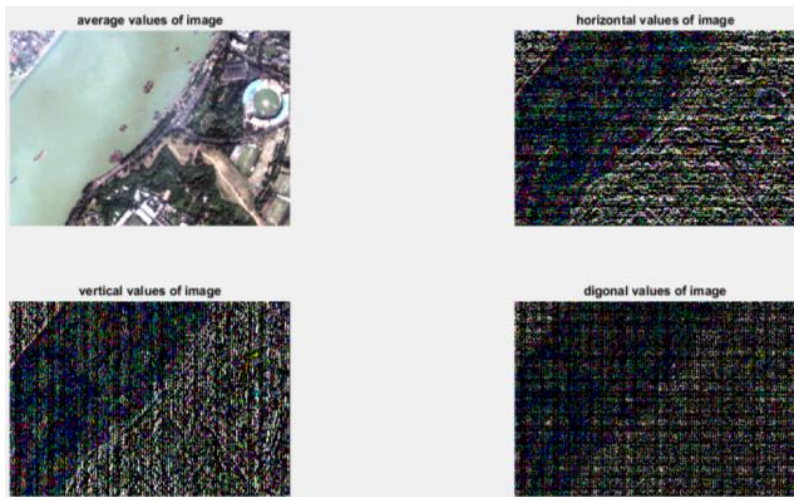

Fig 27: Average, horizontal, vertical, and diagonal value image of first level

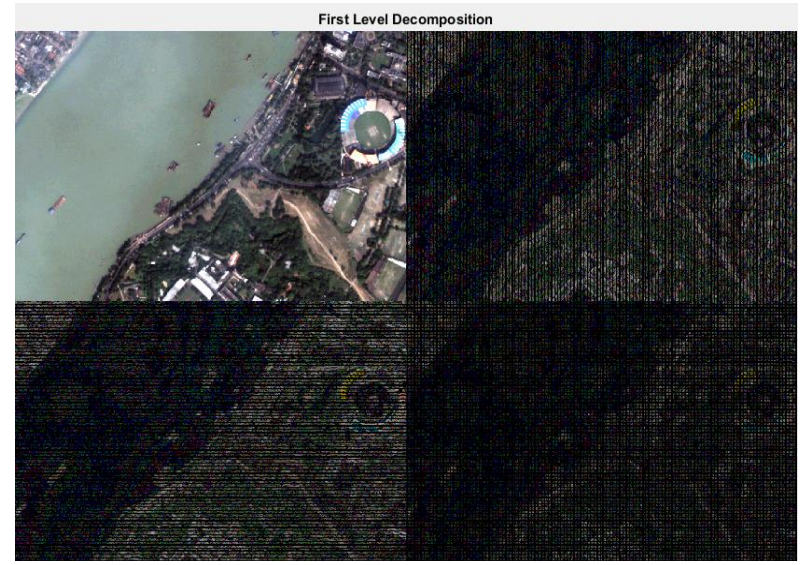

Fig 28: First level Decompoition 

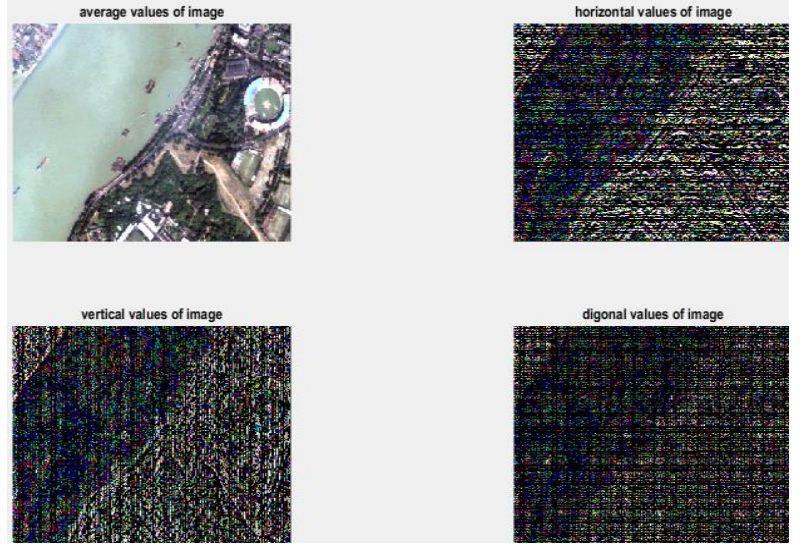

Fig 29: Average, horizontal, vertical, and diagonal value image of second level

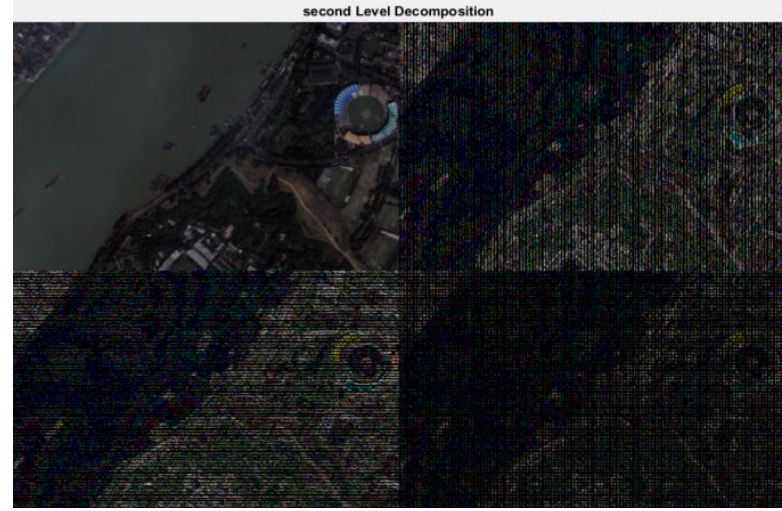

Fig 29 :Second level Decomposition

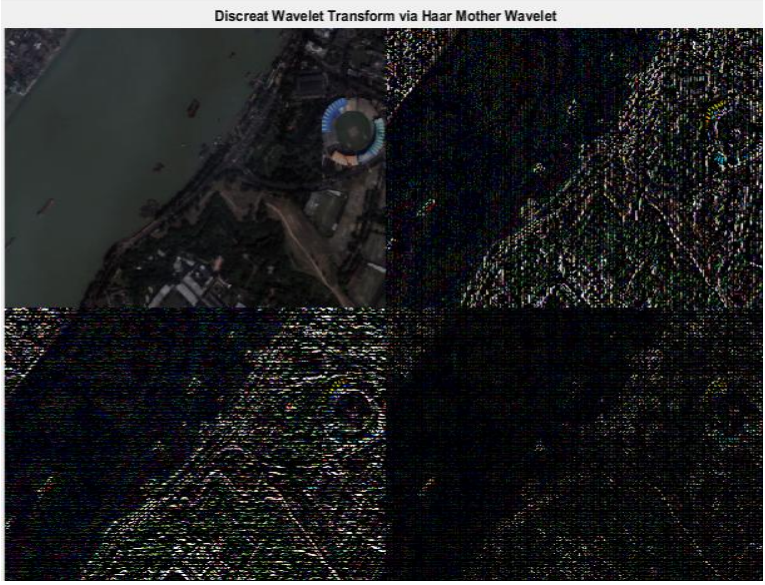

Fig 30: DWT via Haar Mother Wavelet

\subsubsection{Color Enhancement}

Color Enhancement Techniques transform the gray level image into the color image model like RGB, HSI, and CMY. For the satellite imagery, we have focused on two basic techniques to transform the color: Hue, Saturation \& Intensity (HSI) Transform, and Pseudo-Color transforms. [1]

\subsubsection{Hue, saturation and intensity transform}

The HSI model exploits the theory of hue $(\mathrm{H})$, saturation (S) and intensity (I) to illustrate the idea of color. Hue is the dominant wavelength of the color we visualize. It gives unusual colors such as red, green, orange and magenta. The saturation refers to the degree of purity of a color and its diffusion. A pure color is $100 \%$ saturated. Intensity is a measure of the brightness of a color. The HSI color space is popular in Image Enhancement because its visitation is based on human color observation. [1] [10] [11]

The equations for conversion of the image from RGB to HSI are given below:

$$
\begin{aligned}
& I=\frac{1}{3}(R+G+B) \\
& S=1-\frac{3}{(R+G+B)}[\min (R, G, B)]
\end{aligned}
$$

And,

$$
H=\cos ^{-1}\left\{\frac{0.5[(R-G)+(R-B)]}{\sqrt{(R-G)^{2}+(R-B)(G-B)}}\right\}
$$
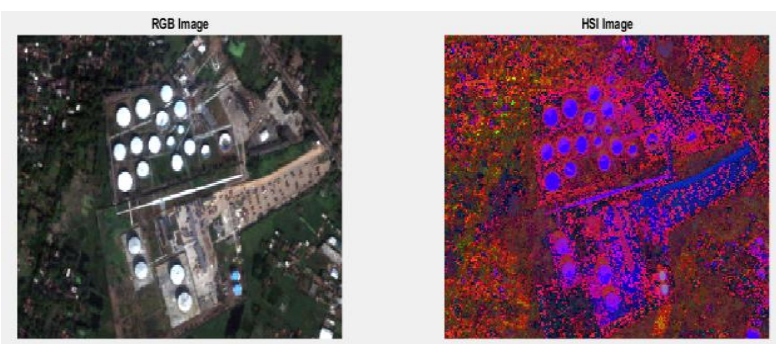

Fig 31: RGB image to HSI image
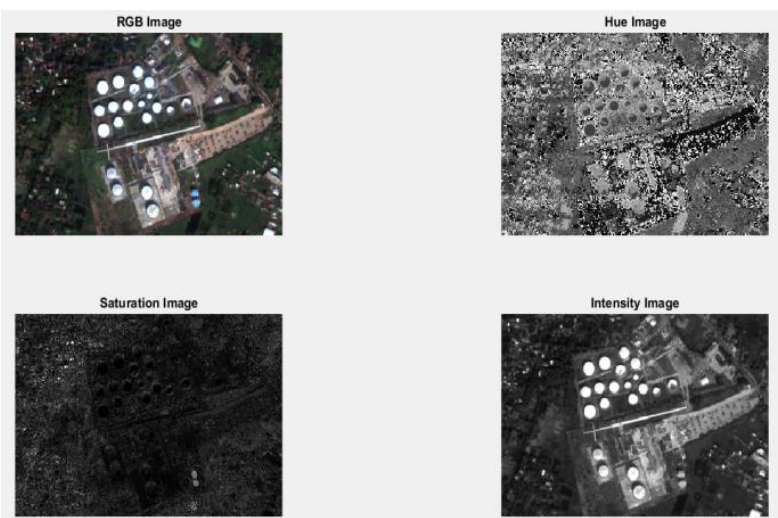

Fig 34: RGB image divided in Hue, Saturation, and Intensity
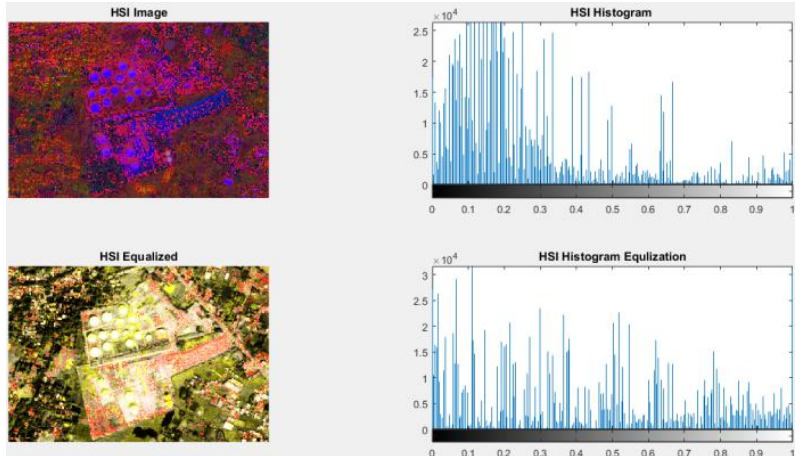

Fig 35: HSI Histogram Equalization Image. 


\subsubsection{Pseudo-color}

Pseudo color maps the one-dimensional gray scale image into intense RGB colored image. Pseudo coloring doesn't boost the image information; it only fixes some details which makes it more visible by increasing the space between successive gray levels. [1] [9] [11]
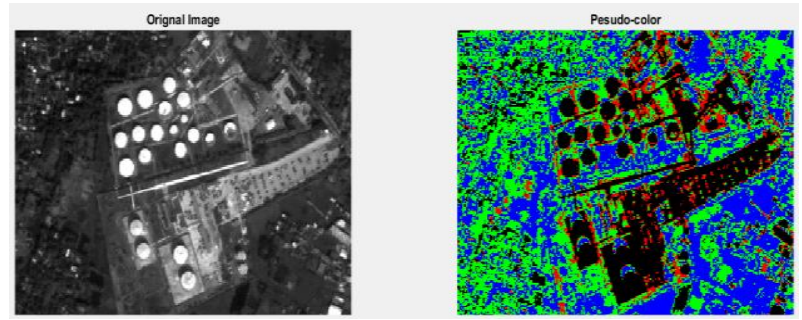

Fig 36: Gray Level Image to Pseudo-Color Transform

\section{ANALYSIS}

\subsection{Visual assessments}

Human eyes are the best example of remote sensing; because it determines the variations of color when it starts visualizing something. Similarly, gray level image illusion's intensity is improvised by color formation in the image. This analysis is done under the grayscale image \& RGB image. We start from a spatial domain which has been studied in three parts. The first part is the low pass filter; the second is the high pass filter and third is the contrast enhancement. We implement LPF with the Gaussian noise to make the image smooth. We have used three different methods to remove Gaussian noise from low pass filter: first is Median, second is Movingaverage and third is Adaptive. We visualize the Median filter noise; as its removing quality is better than the other two filters. It gives a nice smoother image, and the resulting image of an Adaptive filter is more smother than the Moving average filter. In a spatial domain, two methodologies work as HPF (High pass filter) to sharpen the image edges. These are Image Subtraction Method or Derivative based Method. Both methods generate approximately equal sharpen images. In Contrast enhancement methodology, we manipulate the brightness level of the image. Linear contrast Stretch, Histogram equalization, Gaussian Stretch, and Gray Level slicing are the common method of the Contrast Enhancement. Linear contrast stretch is the basic level of the Histogram equalization. HE is the most popular technique of contrast enhancement because it is visually understandable and easy to explain. We apply $\mathrm{HE}$ in different methodology: i.e. in contrast stretching, color HE, and HIS-HE. The contrast stretching-HE is approximately equal to the original equalized image. Color HE makes the image object more visible because it increases the intensity and brightness level of the RGB image. The HSI-HE also increases the brightness level of the HIS image; but some objects hide behind extreme illumination and are not visible clearly, yet it helps us to see the things that have not been previously spotted by other methodology. Gaussian Stretch focus on the extension of distribution i.e. re-introduces the contrast region. To understand this, check the histogram of the Gaussian stretch method. Gray-level slicing increases the gray level of any image. The main drawback of this approach is that it discards the other information of the image.

In a Frequency domain, the image is transformed in sine or cosine waves of different characteristics such as frequency or phase. Then we apply two different approaches: the discrete Fourier transform and discrete wavelet transform. Fourier transform filters are divided into three categories: Low-pass filter, High-pass filter and the notch filter. The first two filters, we have implemented with three different methods: Ideal Filter, Butterworth Filter and the Gaussian Filter. Ideal low pass \& high pass filters are complement to each other. It is practically impossible to implement IHPF. It is visually hard to identify the variation between Butterworth and Gaussian filters; that's why we plot a graph of performance index analysis of image comparison by objective fidelity criteria, which are SNR \& PSNR. This comparison is done under the basis of cutoff value $\mathrm{D}_{0}=10,20,40$, and 60 (fig. $40 \& 41$ ). Notch filter is a partially high-pass filter known as the band stop filter. It sharpens the edges as well as increases the intensity of the objects.

In this paper, we implement two color transforms: Pseudocolor \& HSI color transforms. Pseudocolor- transforms the gray level image into an RGB color image. This helps us to identify the hidden elements of the images and define the populated area or vegetation area. HSI transforms the RGB color image into HSI color format that manipulates the imagery in unusual colors such as red, green, orange and magenta with high intensity. Both techniques make things more visible by increasing the color intensity level.

\subsection{Value analysis}

Performance index analysis of image comparison is done using objective fidelity criteria. These are PSNR (peak signal to noise ratio) and SNR (signal to noise ratio).PSNR uses a more objective and qualitative measurement of distortion. It uses a constant value in which to relate the noise against as an alternative of an instable signal as in SNR.

$P S N R=10 \times \log _{10}\left[\frac{\left(2^{b}-1\right)^{2}}{\frac{1}{M \times N} \sum_{m=0}^{M-1} \sum_{n=0}^{N-1}(f(m, n)-g(m, n))^{2}}\right]$

SNR is used to compare the performance of replicated images, which is defined as

$S N R=10 \times \log _{10}\left[\frac{\frac{1}{M \times N} \sum_{m=0}^{M-1} \sum_{n=0}^{N-1} f(m, n)^{2}}{\frac{1}{M \times N} \sum_{m=0}^{M-1} \sum_{n=0}^{N-1}(f(m, n)-g(m, n))^{2}}\right]$

PNSR \& SNR value tables are merged with graphs.

\section{Spatial Domain Low-pass Filter}

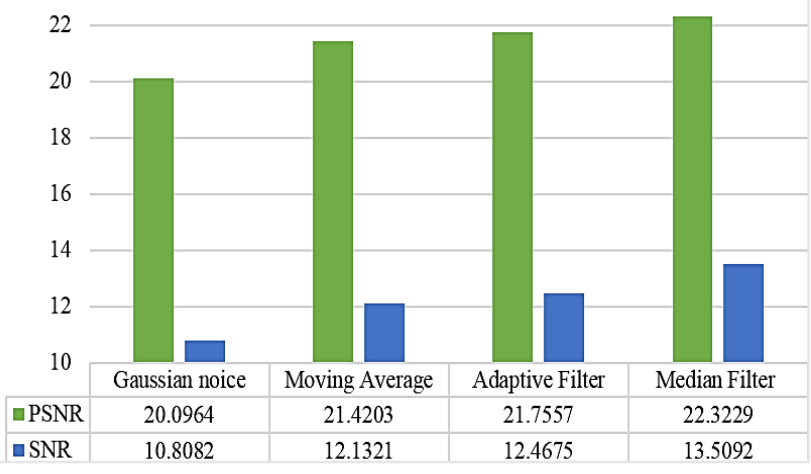

Fig 37: Spatial Domain LPF graph of PSNR \& SNR 


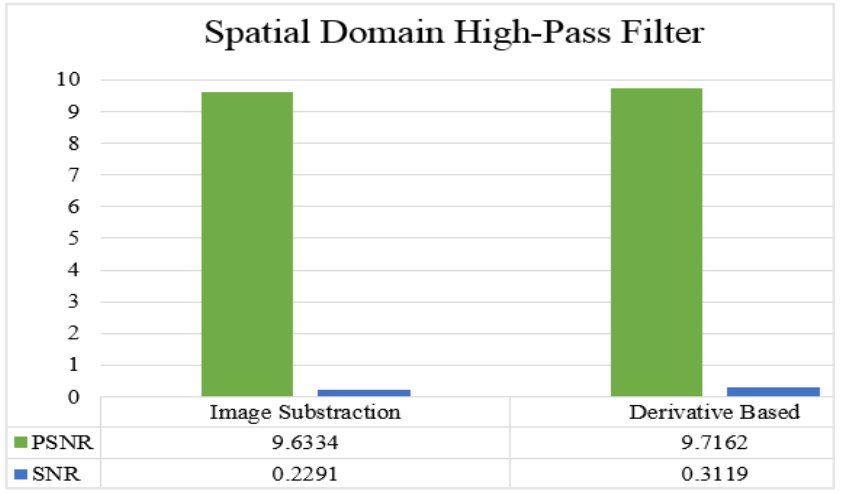

Fig 38: Spatial Domain HPF graph of PSNR \& SNR

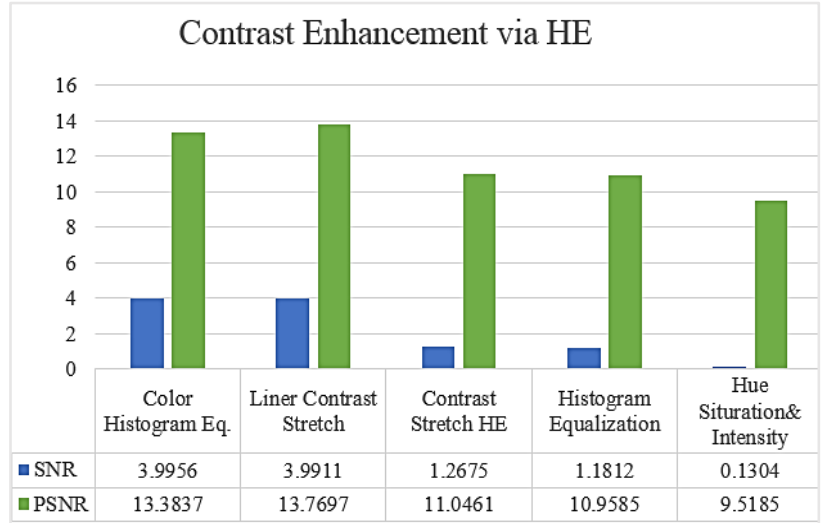

Fig 39: Contrast enhancement graph of PSNR \& SNR

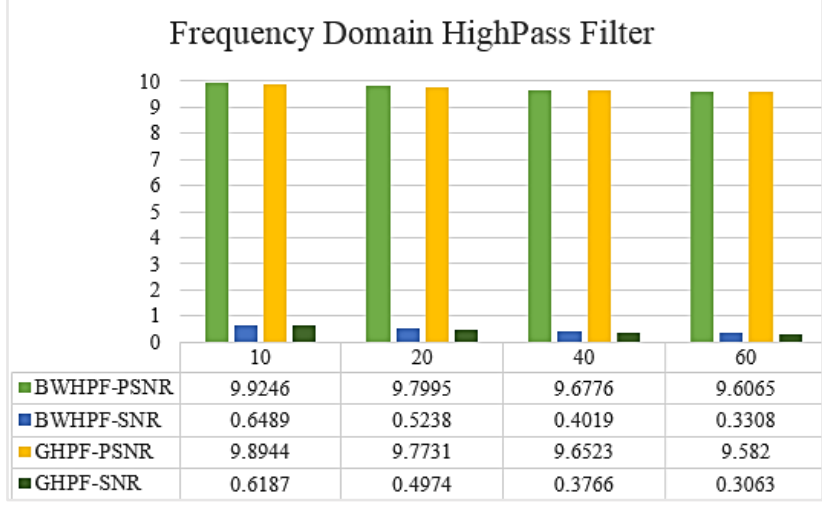

Fig 40: Frequency domain HPF graph of PSNR \& SNR

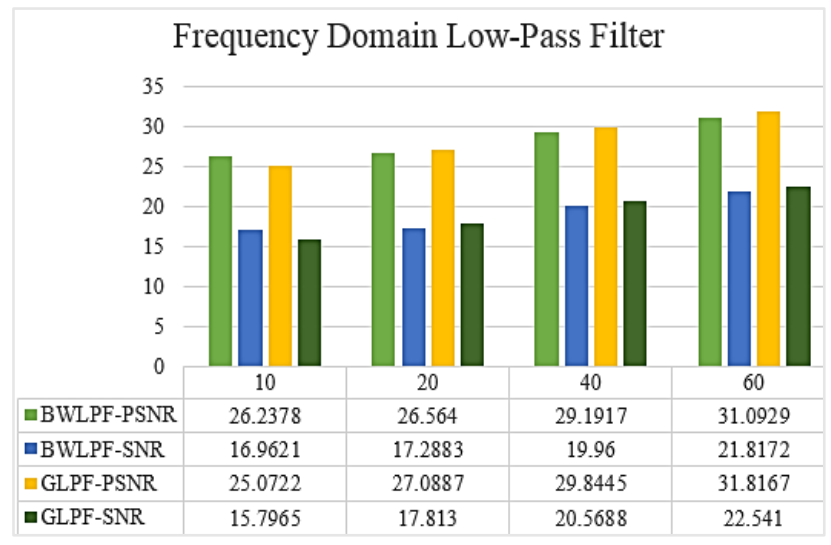

Fig 41: Frequency Domain LPF graph of PSNR \& SNR

\section{CONCLUSION}

In an application area of Satellite Image Enhancement; resolution of the images is not the only problem. We also face equivalent geographical issues such as weather, cloud, fog, and sunlight. They form shadow or a dark zone within an image which is eventually changed with time and atmosphere. That's why we found similar images with minor variations. In the end, we have to visualize and examine the attribute, as a result of the specific need of the image analyst. Identifying the real object and the virtual object from the image is the actual purpose of image enhancement. Our work makes a vision to recognize the effective technique for advance executions like segmentation and classification etc. We tried to cover most of the techniques that are used to enhance the image quality and its attributes. High Pass Filter helps us to identify the edges of the image which are used to define the object of images like elevation area or vegetation area.

\subsection{Future work}

All of these techniques help us to segment an image data and classify image information. This will help us in the future to get more information about the present elements in the Earth and to predict the continuous changes over the Earth. It can be used for better characterization to generate understandable information present in imagery. It can also be used in monitoring and evaluation of the Earth environment.

\section{REFERENCES}

[1] P. M. Mather, Computer Processing of Remotely-Senced Images:An Introduction, third ed., Cited by 2434: Wiley Online Library, 3 April 1999 , p. 350.

[2] R. M. a. H. Aggarwal, "A Comprehensive Review of Image Enhancement Techniques," JOURNAL OF COMPUTING, vol. 2, no. 3, pp. 8-13, March 2010.

[3] Y. B. T. ,. S. I. J. J. Lokare, "Comparative Study of Spatial Domain Image Enhancement Techniques," Journal of Advances in Science and Technology, vol. 12, no. 25, pp. 284 - 287 (4), december 2016.

[4] B. H. Aziz Makandar, "Image Enhancement Techniques using Highpass and Lowpass Filters," International Journal of Computer Applications (0975 - 8887), vol. 109, no. 14, pp. 12-15, January 2015.

[5] A. P. Unni, "Satellite Image Enhancement Using 2D Level DWT," International Journal of Engineering Research \& Technology (IJERT), vol. 3, no. 3, pp. 19261929, March 2014.

[6] A. K. Aditi Sharma, "Satellite Image Contrast and Resolution Enhancement using Discrete Wavelet Transform and Singular Value Decomposition," International Conference on Emerging Trends in Electrical, Electronics and Sustainable Energy Systems (ICETEESES-16) pp. 374-378,Sultanpur, India, 2016.

[7] S. K. Ritika, "Contrast Enhancement Techniques for Images-A Visual Analysis," International Journal of Computer Applications (0975 - 8887), vol. 64, no. 17, pp. 20-25, February 2013.

[8] T. G. Vinod Saini, "A comparative study of image enhancement using Image fusion," International Journal Of Advanced Reserch in Computer Science and Software Engineering, vol. 2, no. 10, pp. 141-145, october 2012. 
[9] S. L. Y. L. K. Y. Zhiwen WANG, "Remote Sensing Image Enhancement Based on Orthogonal Wavelet Transformation Analysis and Pseudo-color Processing," International Journal of Computational Intelligence Systems, vol. 3, no. 6, pp. 745-753, December 2010.

[10] S. E. V. S Jayaraman, Digital Image Processing, vol. 13, The McGraw.Hill companies, 2009.

[11] Mathwork, "Matlab Documentation https://in.mathworks.com/help/matlab/examples.html".

[12] M. M. R. K. H. M. K. a. M. S. Shanto Rahman, "Image Enhancement in Spatial Domain: A Comprehensive Study," International Conference on Computer and Information Technology (ICCIT), vol. 4, no. 17, pp. 369373, 2014.

[13] M. D. H. K. Sawant, "A Comprehensive Review of Image Enhancement Techniques," International Journal of Computer Technology and Electronics Engineering (IJCTEE), vol. 1, no. 2, pp. 39-44.

[14] V. A. S. G. Devarajan, "ANALYSIS OF MEDIAN FILTER".
[15] K.-K. M. How-Lung Eng, "Noise Adaptive SoftSwitching Median Filter," IEEE TRANSACTIONS ON IMAGE PROCESSING, vol. 10, no. 2, pp. 242-251, FEBRUARY 2001.

[16] P. V. S. P. Ahire Rina B., "Overview of Satellite Image Resolution Enhancement Techniques," vol. 3, no. 13, 2013.

[17] P. B.D Jadhav, "An Effective method for Satellite Image Enhancement," International Conferance Computing, Communication and Automation, pp. 1171-1175, 2015.

[18] S. K. Ammu Anna Mathew, "Brightness and Resolution Enhancement of Satellite Images using SVD and DWT," International Journal of Engineering Trends and Technology (IJETT), vol. 4, no. 4, April 2013.

[19] Y. S. R.U Shehorkar, "Resolution Enhancement of Image Captured by Satellite Using DWT," International Journal of Multidisciplinary Research \& Development, vol. 2, no. 7, pp. 238-243, June 2015 . 